\title{
Pharyngeal narrowing in end-stage renal disease: implications for obstructive
} sleep apnoea

\author{
J.M. Beecroft*, V. Hoffstein", A. Pierratos ", C.T. Chan\#, P.A. McFarlane\# and P.J. Hanly*
}

ABSTRACT: Sleep apnoea is common in patients with end-stage renal disease (ESRD). It was hypothesised that this is related to a narrower upper airway. Upper airway dimensions in patients with and without ESRD and sleep apnoea were compared, in order to determine whether upper airway changes associated with ESRD could contribute to the development of sleep apnoea.

An acoustic reflection technique was used to estimate pharyngeal cross-sectional area. Sleep apnoea was assessed by overnight polysomnography. A total of 44 patients with ESRD receiving conventional haemodialysis and 41 subjects with normal renal function were studied. ESRD and control groups were further categorised by the presence or absence of sleep apnoea (apnoea/ hypopnoea index $\geqslant 10$ events $\cdot h^{-1}$ ).

The pharyngeal area was smaller in patients with ESRD compared with subjects with normal renal function: $3.04 \pm 0.84$ versus $3.46 \pm 0.80 \mathrm{~cm}^{2}$ for the functional residual capacity and $1.99 \pm 0.51$ versus $2.14 \pm 0.58 \mathrm{~cm}^{2}$ for the residual volume. The pharynx is narrower in patients with ESRD than in subjects with normal renal function.

In conclusion, since a narrower upper airway predisposes to upper airway occlusion during sleep, it is suggested that this factor contributes to the pathogenesis of sleep apnoea in dialysisdependent patients.

KEYWORDS: Dialysis, kidney failure, pharyngometry, sleep apnoea, upper airway

leep apnoea has been reported in up to $50-70 \%$ of patients with end-stage renal disease (ESRD) [1], a value at least ten times higher than the prevalence reported in the general population [2]. The pathogenesis of sleep apnoea in patients with ESRD remains unclear. Although sleep apnoea is not corrected by conventional haemodialysis or peritoneal dialysis $[3,4]$, it has been reversed both by nocturnal haemodialysis and kidney transplantation [5-7], indicating that its pathophysiology is uniquely associated with the development of chronic renal failure. Previous investigators have observed features of both central and obstructive sleep apnoea (OSA) in patients with ESRD [3-7], which suggests that its pathogenesis is related both to destabilisation of central respiratory control and upper airway occlusion.

In patients without renal failure, the pathogenesis of OSA is associated with anatomical or dynamic narrowing of the upper airway [8, 9]. Individuals with a narrower pharynx are predisposed to upper airway occlusion during sleep and the development of chronic renal failure may create or enhance this in several ways. First, reduced lung volume associated with respiratory muscle weakness or pulmonary oedema can decrease upper airway size [10]. Secondly, both fluid overload and systemic inflammation could cause upper airway oedema and thereby narrow the airway. Thirdly, uremic myopathy or neuropathy involving the upper airway dilator muscles may reduce airway size. Although there is evidence of both sensory and motor neuropathy in the upper airway in patients with OSA and normal renal function [11, 12], this has not been assessed in patients with ESRD.

The objective of the present study was to compare the dimensions of the pharynx in a large group of patients with and without ESRD, further subdivided into individuals with and without sleep apnoea, in order to determine whether ESRD is associated with a narrowed upper airway, which could contribute to the development of sleep apnoea.

\section{METHODS AND MATERIALS \\ Patient recruitment}

All patients receiving conventional haemodialysis (in-centre, 4 h, 3 days week $^{-1}$ ) at the Humber

\section{AFFILIATIONS}

${ }^{*}$ Dept of Medicine, University of Calgary, Calgary, AB,

\#Dept of Medicine, University of Toronto, and

"Dept of Medicine, Humber River Regional Hospital, Toronto, ON, Canada.

CORRESPONDENCE

P.J. Hanly

1421 Health Sciences Centre 3330 Hospital Drive NW

Calgary

Alberta

Canada T2N 4N1

Fax: 14032836151

E-mail: phanly@ucalgary.ca

Received:

December 112006

Accepted after revision:

June 272007

STATEMENT OF INTEREST

None declared 
River Regional Hospital, St. Michael's Hospital and the Toronto General Hospital (all Toronto, ON, Canada), who were referred to the sleep laboratory at St. Michael's Hospital for suspected sleep apnoea, were invited to participate in the present study. A detailed medical history was obtained from each patient, including the cause of renal failure, duration of dialysis treatment, dialysis schedule and medications. A control group, matched for body mass index (BMI) with the ESRD group, was recruited from subjects referred for polysomnography who had no history of kidney disease, cardiovascular dysfunction or upper airway surgery and from healthy volunteers (departmental staff and university students) who had no history of snoring or clinical features of sleep apnoea and were not taking medications that might influence sleep apnoea. The study protocol was reviewed and approved by the research ethics board at St. Michael's Hospital, and all patients gave written informed consent to participate in the study.

\section{Polysomnography}

All patients referred to the sleep laboratory underwent diagnostic polysomnography performed in a standardised fashion in the Sleep Laboratory at St. Michael's Hospital. Healthy volunteers who did not have a history of snoring or clinical features of sleep apnoea did not undergo polysomnography $(n=8)$. Patients with ESRD underwent polysomnography within $24 \mathrm{~h}$ of their last haemodialysis session. Recordings were performed by continuous monitoring of the electroencephalogram (EEG), electrooculogram and sub-mental electromyogram (EMG), ECG, nasal airflow (Ultima Dual Airflow Pressure Sensor; Braebon Medical Corporation, Kanata, ON, Canada), chest and abdominal respiratory movements (Respitrace; Ambulatory Monitoring Inc., Ardsley, NY, USA), oximetry (Mallinckrodt/Nellcor/Puritan-Bennett, Hazelwood, MO, USA) and body position. The recordings were performed and scored by registered polysomnographic technologists according to published criteria [13]. Apnoea was defined as absence of airflow for $>10 \mathrm{~s}$ and hypopnoea was defined as any reduction in airflow for $\geqslant 10 \mathrm{~s}$ associated with an arousal and/or reduction in oxygen saturation $>3 \%$. Apnoeas and hypopnoeas were further classified as: 1) central, if abdominal and ribcage movements were synchronous; 2 ) obstructive, if the movements were paradoxical; and 3) mixed, if a central event had terminal obstructive features. An arousal was defined as a simultaneous increase in alpha activity on the EEG, EMG activation and eye movements which lasted for 3-15 s.

\section{Dialysis adequacy}

A venous blood sample $(3-5 \mathrm{~mL})$ was drawn immediately prior to polysomnography to determine blood urea nitrogen (BUN) and serum creatinine. The percent reduction in urea per dialysis session (PRU) was used to estimate the adequacy of haemodialysis therapy [14] and can be calculated as follows:

$$
\text { PRU }=(\text { pre-BUN-post-BUN }) /(\text { pre-BUN }) \times 100
$$

where pre- and post-BUN are pre- and post-dialysis BUN measurements, and were obtained from the dialysis clinics at the time of polysomnography.

\section{Pharyngometry}

On the evening that polysomnography was performed, an acoustic pharyngometer (Eccovision; Hood Laboratories,
Washington, MA, USA) was used to measure pharyngeal cross-sectional area. The acoustic reflection technique is a noninvasive method for measuring pharyngeal area. It is based on the assumption that the respiratory tract can be modelled as a series of branched tubes of varying cross-sectional area. When a sound wave is sent along such a tract, the wave is partially reflected back every time there is a change in the cross-sectional area of the tract. Measuring the arrival time of these reflections and assuming the speed of sound in the airway, it is possible to calculate the distance travelled by the sound. Knowing the amplitude of the reflected waves, the cross-sectional area of the tube can be calculated. Theoretical considerations and limitations of this method have been described previously $[15,16]$.

Measurements were obtained at the end of a normal tidal breath (functional residual capacity; FRC) and at the end of a forced expiration (residual volume; RV). These measurements were performed during oral breathing; nasal breathing was prevented by nose clips. The pharyngeal cross-sectional area was estimated between the oropharyngeal junction and the glottis (fig. 1). These anatomical landmarks were identified by instructing patients to breathe through the nose, which causes airway narrowing at the oropharyngeal junction, and by performing a Valsalva manoeuvre, which causes airway narrowing at the glottis. The same landmarks were also used to estimate pharyngeal length. Expiratory reserve volume (ERV) was measured using spirometry (Vmax Series 2130 Spirometer; SensorMedics, Yorba Linda, CA, USA). All patients were studied during wakefulness while seated in the upright position with the pharyngometer held in a horizontal position and connected to the patient through a mouthpiece. Subjects were instructed to fix their gaze straight ahead at eye level with head and shoulders aligned in order to avoid excessive head movement. Measurements were taken during four trials at each lung volume (FRC and RV) and the average of these four trials and the coefficient of variation were calculated for each subject. Known sources of artefact,

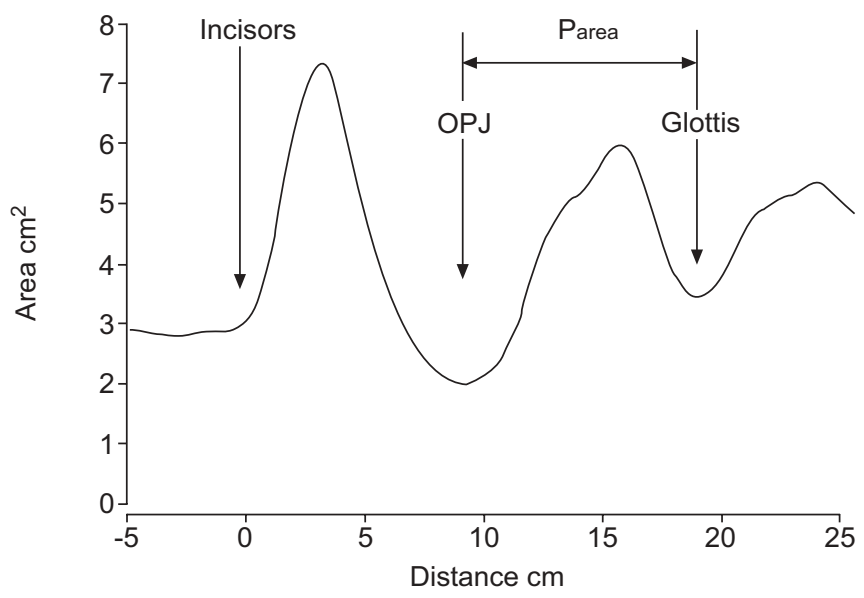

FIGURE 1. Example of a typical pharyngogram. The vertical axis represents the cross-sectional area and the horizontal axis represents the distance into the airway, with $0.0 \mathrm{~cm}$ corresponding to the position of the incisor teeth. The pharyngeal cross-sectional area (Parea) is calculated as the average cross-sectional area between the oropharyngeal junction (OPJ) and the glottis. 


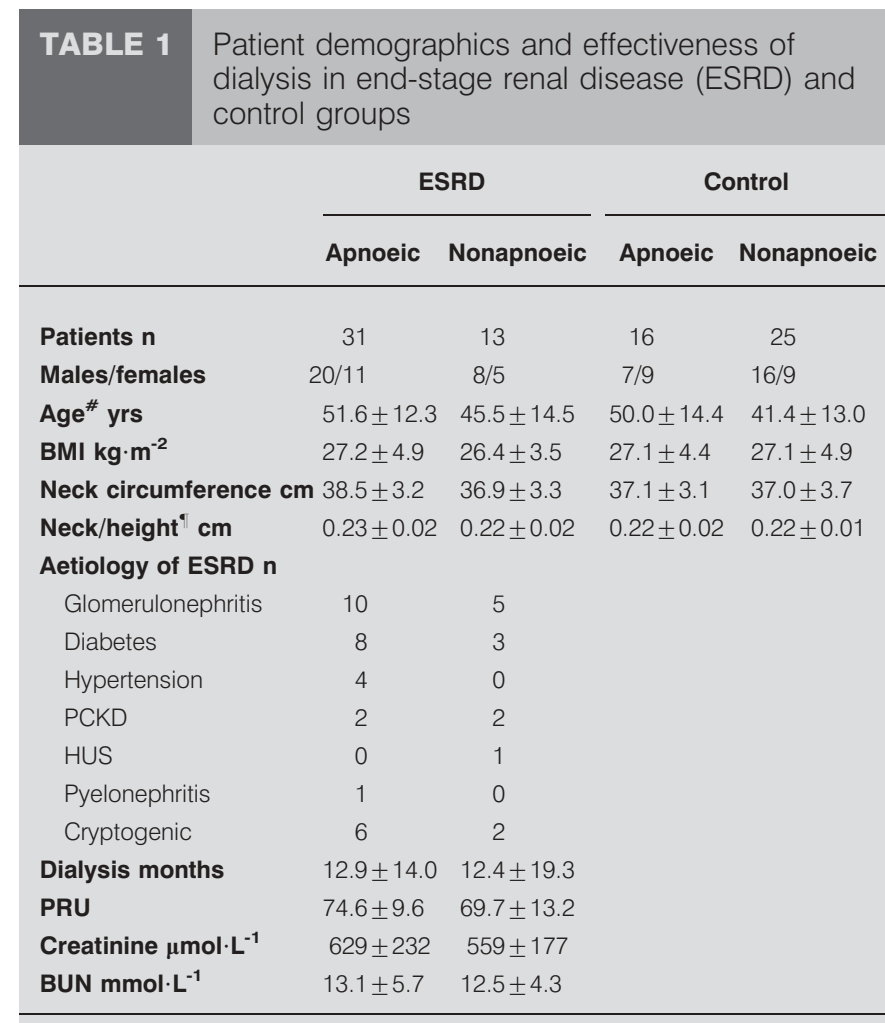

Data are presented as mean \pm SD, unless otherwise stated. BMI: body mass index; PCKD: polycystic kidney disease; HUS: haemolytic uraemic syndrome; PRU: percent reduction in urea per dialysis session; BUN: blood urea nitrogen. \#: $p=0.019$, apnoeic versus nonapnoeic; " : neck circumference indexed to height.

including head extension or flexion and uncontrolled tongue position, were avoided.

\section{Analysis}

Mean data and SDs were analysed using ANOVA, regression analysis and unpaired t-tests. Nominal data were analysed using Chi-squared analysis. All p-values $<0.05$ were considered statistically significant.

\section{RESULTS}

\section{Patient demographics and dialysis adequacy}

A total of 85 patients (51 males and 34 females) were recruited, aged 18-77 yrs (table 1). Patients were divided into ESRD and control groups (normal renal function). Within each group, patients were further classified as apnoeic and nonapnoeic, with sleep apnoea defined as an apnoea/hypopnoea index $(\mathrm{AHI}) \geqslant 10$ events $\cdot \mathrm{h}^{-1}$. There were 44 patients with ESRD and 41 controls. Sex distribution was similar between the groups. Although there were no differences in age between ESRD and control groups, apnoeic patients were significantly older than nonapnoeic patients. By study design, the groups were matched for BMI in order to control for the potential impact of obesity on the upper airway.

The most common cause of ESRD was chronic glomerulonephritis, followed by diabetes mellitus, hypertension, polycystic kidney disease, haemolytic uraemic syndrome and pyelonephritis. In eight patients, the cause of ESRD was unknown. The duration of conventional haemodialysis treatment, as well as the effectiveness of dialysis, reflected by PRU, BUN and serum creatinine, was similar between apnoeic and nonapnoeic patients. Five patients with ESRD were taking benzodiazepines and were instructed to use their medication as usual.

\section{Polysomnography}

The proportion of patients who had sleep apnoea was 71 and $39 \%$ in the ESRD and control groups, respectively. By definition, AHI was significantly higher among apnoeic patients within both ESRD and control groups (table 2), and apnoeas and hypopnoeas were predominantly obstructive, with a smaller proportion classified as central or mixed (table 3$)$. In all patients, the majority $(>65 \%)$ of respiratory events had obstructive features. The frequency of obstructive, central or mixed apnoeas and hypopnoeas did not differ significantly between ESRD and control groups. Mean arterial

TABLE 2 Polysomnography in end-stage renal disease (ESRD) and control groups

\begin{tabular}{|c|c|c|c|c|}
\hline & Apnoeic & Nonapnoeic & Apnoeic & Nonapnoeic ${ }^{\#}$ \\
\hline$A H I^{\star * *}$ events $\cdot h^{-1}$ & $33.6 \pm 22.7$ & $4.5 \pm 3.3$ & $33.1 \pm 16.9$ & $4.7 \pm 3.0$ \\
\hline Mean $\mathrm{Sa}, \mathrm{O}_{2} \%$ & $94.3 \pm 1.9$ & $94.8 \pm 2.2$ & $94.3 \pm 1.5$ & $94.9 \pm 1.4$ \\
\hline Total sleep time $\mathbf{h}$ & $5.3 \pm 1.1$ & $6.0 \pm 1.2$ & $5.8 \pm 1.3$ & $5.1 \pm 1.4$ \\
\hline Stage $2 \%$ total sleep time & $53.2 \pm 13.5$ & $52.7 \pm 9.2$ & $56.9 \pm 12.6$ & $55.8 \pm 12.1$ \\
\hline Slow wave sleep $\%$ total sleep time & $17.6 \pm 8.7$ & $21.0 \pm 10.2$ & $20.0 \pm 9.1$ & $19.2 \pm 14.2$ \\
\hline REM sleep \% total sleep time & $18.7 \pm 8.2$ & $19.2 \pm 10.4$ & $14.6 \pm 6.2$ & $16.6 \pm 5.6$ \\
\hline Total arousals*** events $\cdot h^{-1}$ & $44.0 \pm 27.9$ & $15.5 \pm 11.6$ & $40.2 \pm 13.4$ & $19.1 \pm 9.7$ \\
\hline Awakenings events $\cdot h^{-1}$ & $7.1 \pm 7.0$ & $3.7 \pm 1.8$ & $6.0 \pm 5.0$ & $4.7 \pm 1.7$ \\
\hline
\end{tabular}

Data are presented as mean \pm SD. AHI: apnoea/hypopnoea index; $\mathrm{Sa}_{1} \mathrm{O}_{2}$ : arterial oxygen saturation; REM: rapid eye movement. ${ }^{*}$ : only data for patients who underwent overnight polysomnography are shown; $"$ : total sleep time expressed as a proportion of total study duration. ${ }^{* *}$ : $p<0.001$, apnoeic versus nonapnoeic. 


\begin{tabular}{|c|c|c|c|}
\hline \multirow[t]{2}{*}{ TABLE 3} & \multicolumn{3}{|c|}{$\begin{array}{l}\text { Respiratory events during sleep in patients with } \\
\text { sleep apnoea }\end{array}$} \\
\hline & & ESRD & Control \\
\hline \multicolumn{4}{|l|}{ Obstructive } \\
\hline Events $\cdot h^{-1}$ & & $29.4 \pm 20.7$ & $31.0 \pm 14.9$ \\
\hline Events $\cdot h^{-1} c$ & curring in REM sleep & $39.2 \pm 29.0$ & $43.4 \pm 20.6$ \\
\hline Events $\cdot h^{-1} c$ & curring in non-REM sleep & $27.4 \pm 21.1$ & $28.7 \pm 15.2$ \\
\hline$\%$ of total $€$ & ents & $90.4 \pm 17.9$ & $94.8 \pm 7.0$ \\
\hline \multicolumn{4}{|c|}{ Central } \\
\hline Events $\cdot h^{-1}$ & & $2.4 \pm 4.6$ & $0.8 \pm 1.5$ \\
\hline Events $\cdot h^{-1}$ & ccurring in REM sleep & $0.4 \pm 0.8$ & $0.4 \pm 1.6$ \\
\hline Events $\cdot h^{-1}$ & ccurring in non-REM sleep & $2.8 \pm 5.4$ & $0.9 \pm 1.6$ \\
\hline$\%$ of total $\epsilon$ & ents & $6.4 \pm 10.1$ & $2.6 \pm 4.6$ \\
\hline \multicolumn{4}{|c|}{ Mixed } \\
\hline Events $\cdot h^{-1}$ & & $1.8 \pm 7.2$ & $1.3 \pm 2.8$ \\
\hline Events $\cdot h^{-1}$ & ccurring in REM sleep & $1.1 \pm 5.5$ & $0.8 \pm 2.4$ \\
\hline Events $\cdot h^{-1}$ & ccurring in non-REM sleep & $1.9 \pm 7.4$ & $1.3 \pm 3.0$ \\
\hline$\%$ of total $\epsilon$ & ents & $3.1 \pm 10.4$ & $2.6 \pm 5.5$ \\
\hline \multicolumn{4}{|c|}{ Total } \\
\hline Events $\cdot h^{-1}$ & & $33.6 \pm 22.7$ & $33.1 \pm 16.9$ \\
\hline Events $\cdot h^{-1}$ & ccurring in REM sleep & $40.8 \pm 28.1$ & $44.6 \pm 19.7$ \\
\hline Events $\cdot h^{-1}$ & ccurring in non-REM sleep & $32.1 \pm 23.9$ & $30.9 \pm 17.9$ \\
\hline
\end{tabular}

Data are presented as mean $\pm \mathrm{SD}$. Apnoeas and hypopnoeas were classified as central if abdominal and ribcage movements were synchronous, as obstructive if the movements were paradoxical and as mixed if a central event had terminal obstructive features. ESRD: end-stage renal disease; REM: rapid eye movement.

oxygen saturation was not significantly different between the groups (table 2).

Total sleep time and sleep efficiency were greater in nonapnoeic than apnoeic patients within the ESRD group in contrast to the control group, wherein both total sleep time and sleep efficiency were greater in apnoeic than nonapnoeic patients (table 2). The percentages of rapid eye movement (REM), non-REM and slow wave sleep were similar between groups. Apnoeic patients had a significantly greater number of

\begin{tabular}{|c|c|c|c|c|c|}
\hline \multirow[t]{3}{*}{ TABLE 4} & \multicolumn{5}{|c|}{$\begin{array}{l}\text { Pharyngometry in end-stage renal disease } \\
\text { (ESRD) and control groups }\end{array}$} \\
\hline & & \multicolumn{2}{|c|}{ ESRD } & \multicolumn{2}{|c|}{ Control } \\
\hline & & Apnoeic & Nonapnoeic & Apnoeic & Nonapnoeic \\
\hline \multicolumn{6}{|c|}{$\begin{array}{l}\text { Pharyngeal area } \\
\mathrm{cm}^{2}\end{array}$} \\
\hline FRC & & $3.10 \pm 0.92$ & $2.91 \pm 0.63$ & $3.23 \pm 0.82$ & $3.60 \pm 0.77$ \\
\hline $\mathrm{RV}$ & & $2.00 \pm 0.55$ & $1.98 \pm 0.41$ & $2.16 \pm 0.67$ & $2.12 \pm 0.54$ \\
\hline \multirow{2}{*}{\multicolumn{2}{|c|}{ Pharyngeal length $\mathrm{cm}$}} & $9.28 \pm 1.49$ & $8.90 \pm 1.47$ & $9.37 \pm 1.77$ & $9.52 \pm 1.65$ \\
\hline & & $0.94 \pm 0.55$ & $1.02 \pm 0.52$ & $1.15 \pm 0.49$ & $1.45 \pm 0.51$ \\
\hline
\end{tabular}

Data are presented as mean \pm SD. FRC: functional residual capacity; RV: residual volume; ERV: expiratory reserve volume. ${ }^{* *}: p<0.001$, FRC versus RV; \#: $p=0.033$, ESRD versus control; $\because p=0.014$, ESRD versus control. arousals from sleep, which were predominantly arousals associated with apnoeas and hypopnoeas. There were no significant differences in the number of arousals and awakenings between ESRD and control groups. The number of awakenings was not significantly different between groups.

\section{Pharyngometry}

Within all groups, the pharyngeal cross-sectional area was significantly greater at FRC than RV (table 4). The pharyngeal cross-sectional area was significantly smaller both at FRC and $\mathrm{RV}$ in patients with ESRD compared with controls $(3.04 \pm 0.84$ versus $3.46 \pm 0.80 \mathrm{~cm}^{2}$ for FRC and $1.99 \pm 0.51$ versus $2.14 \pm 0.58 \mathrm{~cm}^{2}$ for $\mathrm{RV}$; mean difference $0.28 \pm 0.13,95 \%$ confidence interval $0.02-0.54 ; \mathrm{p}=0.033)$. This difference remained significant when data from patients receiving benzodiazepines were excluded. However, the pharyngeal cross-sectional area was not different between apnoeic and nonapnoeic patients $\left(3.15 \pm 0.88\right.$ versus $3.36 \pm 0.79 \mathrm{~cm}^{2}$ for FRC and $2.05 \pm 0.59$ versus $2.07 \pm 0.50 \mathrm{~cm}^{2}$ for $\mathrm{RV}$; mean difference $0.03 \pm 0.13,95 \%$ confidence interval $-0.23-0.29)$. Mean intrasubject coefficient of variation, measured at FRC, was acceptably low ( $\pm \pm 4 \%$ for all subjects) and was similar between ESRD and control groups $(5 \pm 4$ and $5 \pm 5 \%$, respectively). There were no significant intergroup differences in pharyngeal length. ERV was similar between patients with and without sleep apnoea, but significantly smaller in patients with ESRD compared with controls $(0.96 \pm 0.53$ versus $1.33 \pm 0.52 \mathrm{~L}$; $p=0.014)$. Within the ESRD group, there was a significant positive correlation between ERV and pharyngeal crosssectional area at FRC $(\mathrm{r}=0.492, \mathrm{p}=0.001)$ and $\mathrm{RV}(\mathrm{r}=0.363$, $\mathrm{p}=0.019)$.

\section{DISCUSSION}

Previous studies in the nonrenal failure population have found a narrower upper airway in patients with OSA compared with healthy subjects $[8,9,17,18]$. Narrowing of the upper airway increases the likelihood of upper airway occlusion during sleep, when diminished dilator muscle tone and gravitational forces associated with the supine position combine to narrow the airway further. Previous investigators [17], using the acoustic reflection technique, reported that the pharyngeal cross-sectional area was $26 \%$ smaller in patients with OSA, than in those without OSA. It was found that the pharyngeal cross-sectional area in patients with ESRD was $12 \%$ smaller than in non-ESRD control subjects. Recent work using the same methodology as in the present study [19] has shown that increasing the pharyngeal cross-sectional area as little as $6 \%$, concurrent with weight loss, was sufficient to reduce the severity of sleep apnoea, as reflected by the AHI, by $73 \%$. Consequently, it is believed that the difference in crosssectional area found between patients with ESRD and subjects with normal renal function is clinically significant, and may contribute to the development of sleep apnoea in this patient population. Pharyngeal narrowing in nonrenal failure patients with OSA may be associated with increased para-pharyngeal fat due to obesity, and thickened lateral pharyngeal walls possibly due to genetic inheritance or trauma associated with recurrent intraluminal negative pressure swings and snoring vibration. Upper airway calibre is also influenced by sex and age $[20,21]$. However, these mechanisms do not explain the present findings, since there were no significant differences in 
BMI, age, sex distribution or severity of sleep apnoea between patients with ESRD and with normal renal function (tables 1, 2 and 4). Consequently, it is possible to speculate on potential reasons for upper airway narrowing that are unique to haemodialysis patients.

Upper airway size is significantly altered by changes in lung volume: widening as lung volume increases and narrowing as lung volume falls [10]. Consequently, pharyngeal narrowing in ESRD patients may be related to reduced lung volume, as reflected by the smaller observed ERV (table 4). Respiratory muscle weakness has been described in patients with ESRD [22], which could decrease FRC by reducing chest wall expansion. Alternatively, FRC may be reduced by pulmonary oedema associated with fluid overload, which is common in ESRD. Decreased lung volume associated with respiratory muscle weakness and/or fluid overload can increase upper airway collapsibility and thereby reduce upper airway calibre by reducing caudal tracheal traction $[23,24]$. These possibilities are supported by the positive correlation found between ERV and pharyngeal cross-sectional area in patients with ESRD. However, the change in ERV accounted for $<25 \%$ of the variability in pharyngeal cross-sectional area, which indicates that other potential mechanisms for reduced pharyngeal size must be considered.

Increased vascular distension in the upper airway due to fluid overload could contribute to pharyngeal narrowing in ESRD. In healthy subjects, decreasing central venous pressure by impeding venous return to the heart using leg cuff inflation increased upper airway dimensions [25]. Fluid overload could also lead to interstitial oedema of the pharyngeal wall or parapharyngeal tissues, which can narrow the airway. This suggestion is supported by the recent report that fluid displaced rostrally from the legs increases pharyngeal resistance in healthy subjects [26]. Alternatively, upper airway oedema and pharyngeal narrowing could be caused by systemic inflammation. Pharyngeal narrowing has been noted in pre-eclampsia [27], which may be related to widespread systemic inflammation and oedema. ESRD is a chronic inflammatory state and similar mechanisms may contribute to pharyngeal narrowing in this patient population. Another possibility is upper airway dilator muscle dysfunction due to neuropathy or myopathy associated with chronic uraemia, or to the underlying cause of ESRD, such as diabetes mellitus. Sensory neuropathy has been demonstrated in the upper airway of OSA patients with normal renal function [12] and may exacerbate the disease process: topical anaesthesia of the upper airway increases apnoea duration in patients with OSA [28]. Muscle denervation has been described in nonrenal failure patients with OSA [11], and may contribute to upper airway narrowing.

Although it was found that the pharyngeal cross-sectional area was smaller in patients with ESRD than in control subjects, it was not significantly different between those with and without OSA. These findings are not unique. STAUfFER et al. [29] measured the pharyngeal cross-sectional area using computerised tomography and found no difference between males with and without OSA matched for age and BMI. Similarly to the present study, the authors included both snoring and nonsnoring subjects in the control group. Inclusion of snorers in the present control group may account for the similarities observed in pharyngeal size between apnoeic and nonapnoeic patients, as there is evidence that the pharyngeal crosssectional area is similar between nonapnoeic snorers and patients with OSA [17]. Conflicting results between studies may also be related to differences in the measurement technique. Acoustic reflection measures the cross-sectional area from the oropharyngeal junction to the glottis, but it does not measure the dimensions of the velopharynx or the shape or configuration of pharyngeal structures. More recent studies [9, 18] have noted differences in the velopharyngeal crosssectional area between patients with and without OSA, but have failed to find differences in the size of the oropharynx. Differences in the configuration of the velopharynx (lateral narrowing), but not the oropharynx, also distinguished apnoeic from nonapnoeic patients [18]. The relevance of these findings with regard to the present results is highlighted by the observation that the velopharynx has been identified as the primary site of occlusion in patients with OSA [9].

Notwithstanding these potential explanations for the absence of significant differences between apnoeic and nonapnoeic patients, the present findings suggest that pharyngeal narrowing alone does not account for the development of sleep apnoea in patients with ESRD, and that interaction with another pathogenic factor is required. It is not believed that the presence of sleep apnoea in ESRD patients in the present study was due to the timing of dialysis, since polysomnographic and respiratory assessments in ESRD patients were performed in a standardised fashion (within $24 \mathrm{~h}$ of the last haemodialysis session). Furthermore, the development of sleep apnoea was not related to the variability in the efficiency of haemodialysis, since no difference was found in PRU, BUN or serum creatinine between ESRD patients with and without sleep apnoea. It is possible that the development of sleep apnoea in the present patient population depends on the interaction between upper airway narrowing and the stability of ventilatory control [30]. There is evidence that instability in central control of respiration can be associated with upper airway occlusion during sleep. In an experimental model of central control instability induced by transient hypoxia during sleep [31], the likelihood that central instability was accompanied by upper airway closure was greater if the airway was narrow. More recently, it has been reported that increased loop gain, which reflects ventilatory instability, is associated with the development of OSA in patients whose upper airway closes during sleep at a near-zero luminal pressure [32]. Increased respiratory chemoreflex responsiveness in patients with ESRD and OSA has been previously observed [33], a factor which is known to destabilise central respiratory control. It is possible that the combination of central destabilisation and upper airway narrowing contributes to the development of OSA in patients with ESRD.

The present study has some limitations. First, upper airway measurements were performed during wakefulness while other respiratory measurements were performed during sleep. The current authors acknowledge that sleep onset induces changes in the upper airway that are pivotal to the development of OSA. However, it is believed that the pharyngeal narrowing observed during wakefulness continued during sleep and contributed to the pathogenesis of sleep apnoea in 
patients with ESRD. Secondly, acoustic reflection measurements in the upper airway vary significantly between individuals, which can make it difficult to find significant differences between groups of subjects. The comparison between groups may be limited by a lack of overall power, attributed in part to this inherent variability and also to the small sample size. Nevertheless, the fact that significant differences were found between patients with and without ESRD, despite the inherent variability in the measurement, makes the present findings more robust. Finally, the present study design was cross-sectional and consequently cannot infer causality between pharyngeal changes and sleep apnoea in patients with ESRD. Nevertheless, it is believed that pharyngeal narrowing is likely to contribute to upper airway occlusion during sleep, particularly when it is combined with other factors, such as instability in central respiratory motor output.

In summary, the pharyngeal cross-sectional area is reduced in patients with end-stage renal disease. Since a narrower upper airway predisposes patients to upper airway occlusion during sleep, it is suggested that this factor contributes to the pathogenesis of sleep apnoea in end-stage renal disease. Further studies are required to understand how upper airway narrowing develops and to determine what additional mechanisms contribute to the development of sleep apnoea in patient populations such as the present one.

\section{ACKNOWLEDGEMENTS}

The authors would like to thank J. Gabor for his technical assistance, and S. Crombach, J. Zaltzman and R. Prasad (all University of Toronto, Toronto, ON, Canada) for their assistance with patient recruitment.

\section{REFERENCES}

1 Kraus MA, Hamburger RJ. Sleep apnea in renal failure. Adv Perit Dial 1997; 13: 88-92.

2 Young T, Palta M, Dempsey J, Skatrud J, Weber S, Badr S. The occurrence of sleep-disordered breathing among middle-aged adults. N Engl J Med 1993; 328: 1230-1235.

3 Mendelson WB, Wadhwa NK, Greenberg HE, Gujavarty K, Bergofsky E. Effects of hemodialysis on sleep apnea syndrome in end-stage renal disease. Clin Nephrol 1990; 33: 247-251.

4 Wadhwa NK, Mendelson WB. A comparison of sleepdisordered respiration in ESRD patients receiving hemodialysis and peritoneal dialysis. Adv Perit Dial 1992; 8: 195-198.

5 Auckley DH, Schmidt-Nowara W, Brown LK. Reversal of sleep apnea hypopnea syndrome in end-stage renal disease after kidney transplantation. Am J Kidney Dis 1999; 34: 739-744.

6 Hanly PJ, Pierratos A. Improvement of sleep apnea in patients with chronic renal failure who undergo nocturnal hemodialysis. N Engl J Med 2001; 344: 102-107.

7 Langevin B, Fouque D, Léger P, Robert D. Sleep apnea syndrome and end-stage renal disease. Cure after renal transplantation. Chest 1993; 103: 1330-1335.

8 Haponik EF, Smith PL, Bohlman ME, Allen RP, Goldman SM, Bleecker ER. Computerized tomography in obstructive sleep apnea. Correlation of airway size with physiology during sleep and wakefulness. Am Rev Respir Dis 1983; 127: 221-226.

9 Isono S, Remmers JE, Tanaka A, Sho Y, Sato J, Nishino T. Anatomy of pharynx in patients with obstructive sleep apnea and in normal subjects. J Appl Physiol 1997; 82: 1319-1326.

10 Hoffstein V, Zamel N, Phillipson EA. Lung volume dependence of pharyngeal cross-sectional area in patients with obstructive sleep apnea. Am Rev Respir Dis 1984; 130: 175-178.

11 Boyd JH, Petrof BJ, Hamid Q, Fraser R, Kimoff RJ. Upper airway muscle inflammation and denervation changes in obstructive sleep apnea. Am J Respir Crit Care Med 2004; 170: 541-546.

12 Kimoff RJ, Sforza E, Champagne V, Ofiara L, Gendron D. Upper airway sensation in snoring and obstructive sleep apnea. Am J Respir Crit Care Med 2001; 164: 250-255.

13 Rechtschaffen A, Kales A. A Manual of Standardized Terminology, Techniques and Scoring System for Sleep Stages of Human Subjects. Los Angeles, UCLA Brain Information Service/Brain Research Institute, 1968. NIH publication no. 204.

14 Jindal KK, Manuel A, Goldstein MB. Percent reduction in blood urea concentration during hemodialysis (PRU). A simple and accurate method to estimate Kt/V urea. ASAIO Trans 1987; 33: 286-288.

15 Fredberg JJ, Wohl ME, Glass GM, Dorkin HL. Airway area by acoustic reflections measured at the mouth. J Appl Physiol 1980; 48: 749-758.

16 Hoffstein V, Fredberg JJ. The acoustic reflection technique for non-invasive assessment of upper airway area. Eur Respir J 1991; 4: 602-11.

17 Bradley TD, Brown IG, Grossman RF, et al. Pharyngeal size in snorers, nonsnorers, and patients with obstructive sleep apnea. N Engl J Med 1986; 315: 1327-1331.

18 Ciscar MA, Juan G, Martínez V, et al. Magnetic resonance imaging of the pharynx in OSA patients and healthy subjects. Eur Respir J 2001; 17: 79-86.

19 Busetto L, Enzi G, Inelmen EM, et al. Obstructive sleep apnea syndrome in morbid obesity: effects of intragastric balloon. Chest 2005; 128: 618-623.

20 Brown IG, Zamel N, Hoffstein V. Pharyngeal crosssectional area in normal men and women. J Appl Physiol 1986; 61: 890-895.

21 Martin SE, Mathur R, Marshall I, Douglas NJ. The effect of age, sex, obesity and posture on upper airway size. Eur Respir J 1997; 10: 2087-2090.

22 Bark H, Heimer D, Chaimovitz C, Mostoslovski M. Effect of chronic renal failure on respiratory muscle strength. Respiration 1988; 54: 153-161.

23 Kairaitis K, Byth K, Parikh R, Stavrinou R, Wheatley JR, Amis TC. Tracheal traction effects on upper airway patency in rabbits: the role of tissue pressure. Sleep 2007; 30: 179-186.

24 Thut DC, Schwartz AR, Roach D, Wise RA, Permutt S, Smith PL. Tracheal and neck position influence upper airway airflow dynamics by altering airway length. J Appl Physiol 1993; 75: 2084-2090.

25 Shepard JW, Pevernagie DA, Stanson AW, Daniels BK, Sheedy PF. Effects of changes in central venous pressure on upper airway size in patients with obstructive sleep apnea. Am J Respir Crit Care Med 1996; 153: 250-254. 
26 Chiu KL, Ryan CM, Shiota S, et al. Fluid shift by lower body positive pressure increases pharyngeal resistance in healthy subjects. Am J Respir Crit Care Med 2006; 174: 1378-1383.

27 Izci B, Riha RL, Martin SE, et al. The upper airway in pregnancy and pre-eclampsia. Am J Respir Crit Care Med 2003; 167: 137-140.

28 Cala SJ, Sliwinski P, Cosio MG, Kimoff RJ. Effect of topical upper airway anesthesia on apnea duration through the night in obstructive sleep apnea. J Appl Physiol 1996; 81: 2618-2626.

29 Stauffer JL, Zwillich CW, Cadieux RJ, et al. Pharyngeal size and resistance in obstructive sleep apnea. Am Rev Respir Dis 1987; 136: 623-627.
30 Younes M, Ostrowski M, Thompson W, Leslie C, Shewchuk W. Chemical control stability in patients with obstructive sleep apnea. Am J Respir Crit Care Med 2001; 163: 1181-1190.

31 Warner G, Skatrud JB, Dempsey JA. Effect of hypoxiainduced periodic breathing on upper airway obstruction during sleep. J Appl Physiol 1987; 62: 2201-2211.

32 Wellman A, Jordan AS, Malhotra A, et al. Ventilatory control and airway anatomy in obstructive sleep apnea. Am J Respir Crit Care Med 2004; 170: 1225-1232.

33 Beecroft J, Duffin J, Pierratos A, Chan CT, McFarlane P, Hanly PJ. Enhanced chemo-responsiveness in patients with sleep apnoea and end-stage renal disease. Eur Respir J 2006; 28: 151-158. 